\title{
Spectral pictures of 2-variable weighted shifts *
}

\author{
Raúl E. Curto $^{\mathrm{a}}$, Jasang Yoon ${ }^{\mathrm{b}}$ \\ ${ }^{a}$ Department of Mathematics, The University of Iowa, Iowa City, Iowa 52242 \\ ${ }^{\mathrm{b}}$ Department of Mathematics, Iowa State University, Ames, Iowa 50011
}

\begin{abstract}
We study the spectral pictures of (jointly) hyponormal 2-variable weighted shifts with commuting subnormal components. By contrast with all known results in the theory of subnormal single and 2-variable weighted shifts, we show that the Taylor essential spectrum can be disconnected. We do this by obtaining a simple sufficient condition that guarantees disconnectedness, based on the norms of the horizontal slices of the shift. We also show that for every $k \geq 1$ there exists a $k$-hyponormal 2-variable weighted shift whose horizontal and vertical slices have 1- or 2-atomic Berger measures, and whose Taylor essential spectrum is disconnected.
\end{abstract}

\section{Résumé}

Les images spectrales de shifts pondérés à 2-variables. Nous étudions les images spectrales de shifts pondérés à deux variables et (conjointement) hyponormaux possédant des composants commutants sousnormaux. À la différence de tous les résultats connus dans la théorie des shifts pondérés simples sousnormaux à deux variables, nous démontrons que le spectre essentiel de Taylor peut être déconnecté. Nous faisons cela en obtenant une condition suffisante simple qui garantit le caractère déconnecté de ce spectre, basée sur les normes des sections horizontales du shift. Nous montrons également que pour chaque $k \geq 1$ il existe un shift pondéré $k$-hyponormal à deux variables dont le spectre essentiel de Taylor est déconnecté. Pour citer cet article : A. Name1, A. Name2, C. R. Acad. Sci. Paris, Ser. I 340 (2005).

ऋ Research partially supported by NSF Grants DMS-0099357 and DMS-0400741.

Email addresses: rcurto@math.uiowa.edu (Raúl E. Curto), jyoon@iastate.edu (Jasang Yoon). 


\section{Version française abrégée}

Le Problème du Relèvement des Sousnormaux Commutants (PRSC) demande quelles sont les conditions nécessaires et suffisantes pour qu'une paire commutante d'opérateurs sousnormaux dans l'espace de Hilbert $\mathcal{H}$ admette une paire commutante d'extensions normales agissant sur un espace Hilbert $\mathcal{K} \supseteq \mathcal{H}$. Dans nos recherches récentes nous avons montré que l'hyponormalité, bien que nécessaire, n'est pas une condition suffisante pour le relèvement. Nous avons fait cela en considérant des shifts pondérés commutants en deux variables, pour lesquels nous avons développé de nouvelles techniques pour détecter leur hyponormalité et sousnormalité. À l'intérieur de la classe $\mathfrak{H}_{0}$ de shifts pondérés de 2 -variables $\mathbf{T} \equiv\left(T_{1}, T_{2}\right)$ possédant des composants sousnormaux $T_{1}$ et $T_{2}$, nous avons obtenu dans [12] une nouvelle condition nécessaire pour l'existence d'un relèvement: les mesures de Berger des sections horizontales et verticales doivent être ordonnées de façon linéaire par rapport à la continuité absolue. Plus récemment, dans [5] nous avons donné une solution abstraite du PRSC, après avoir démontré une version multivariée du critère de Bram-Halmos [1].

Dans ce travail, nous développons des techniques et des outils nouveaux, et nous les combinons avec ceux dans [11], [12], [13], [5], [9] et [10], afin d'éclairer la théorie spectrale des shifts pondérés à 2-variables. Comme il est déja bien connu, l'image spectrale d'un shift pondéré unilatéral et hyponormal $W_{\alpha}$ est facile à décrire: le spectre est un disque fermé de rayon $\left\|W_{\alpha}\right\|$, le spectre essentiel est un cercle de rayon $\left\|W_{\alpha}\right\|$, et l'indice de Fredholm est -1 dans le disque ouvert. Ainsi, d'un point de vue spectral tout shift pondéré unilatéral hyponormal de norme 1 est équivalent au shift unilatéral (non-pondéré) $U_{+}$(qui est aussi sousnormal). Pour les shifts pondérés à 2-variables la situation est tout à fait différente, et une description complète de l'image spectrale a été donnée dans [9] et [10], en utilisant la construction du groupoïde introduite dans [16] et [15], et raffinée dans [6]. La présence de la mesure de Berger était essentielle dans l'étude du comportement asymptotique des suites de poids, et a mené à des résultats concrets sur les diverses parties du spectre de Taylor. Pour les shifts pondérés hyponormaux à 2-variables $\mathbf{T} \in \mathfrak{H}_{0}$, pourtant, l'étude des propriétés spectrales exige des techniques indépendantes, puisque aucune mesure de Berger n'est présente. Dans ce qui

suit, nous présentons nombre de résultats qui mettent en relief les différences profondes entre le cas en une variable et celui en deux variables. Dans le Théorème 2.2 nous exhibons, pour la première fois, une condition suffisante qui garantit le caractère déconnecté du spectre essentiel de Taylor, notamment, $\left\|W_{\alpha^{(1)}}\right\|<\left\|W_{\alpha^{(0)}}\right\|$, où $W_{\alpha^{(j)}}$ dénote le $j$-ème niveau horizontal de $T_{1}$; dans l'Exemple 2.4 nous montrons que cette condition peut être présente même dans les paires hyponormales avec des sections $W_{\alpha^{(j)}}(j \geq 1)$ mutuellement absolument continues. Nous améliorons ce résultat en montrant dans le Théorème 2.6 qu'il est possible de former davantage de composantes connexes du spectre essentiel de Taylor, tout en préservant son caractère hyponormal, si nous utilisons des shifts pondérés de type Bergman dans chaque niveau horizontal de $T_{1}$. Ce fait est tout-à-fait étonnant, à la lumière des résultats bien connus sur les shifts pondérés en une variable. Au cours de notre analyse, nous démontrons que pour les shifts pondérés sousnormaux en deux variables, les mesures de Berger des sections 
horizontales $\left\{W_{\alpha^{(j)}}\right\}_{j=1}^{\infty}$ sont toutes mutuellement absolument continues et, par conséquent, $\left\|W_{\alpha^{(j)}}\right\|=\left\|W_{\alpha^{(1)}}\right\|(j \geq 1)$. Cette nouvelle condition nécessaire est facilement calculable, et complète la condition nécessaire précédente qui se trouve dans [12, Theorem 3.3]. Ensuite, nous présentons une application à la théorie des opérateurs à une variable (Exemple 2.8). Finalement, le Théorème 2.10 montre que même si nous supposons que les mesures de Berger des shifts associés aux lignes horizontales et colonnes verticales sont discrètes, la $k$-hyponormalité conjointe [5] de $\mathbf{T}$ (pour un $k \geq 1$ arbitraire) n'est pas une condition suffisante pour forcer l'égalité $\left|\sigma_{T e}(\mathbf{T})\right|=\partial\left|\sigma_{T}(\mathbf{T})\right|$.

\section{Introduction}

The Lifting Problem for Commuting Subnormals (LPCS) asks for necessary and sufficient conditions for a commuting pair of subnormal operators on Hilbert space $\mathcal{H}$ to admit a commuting pair of normal extensions acting on a Hilbert space $\mathcal{K} \supseteq \mathcal{H}$. In recent work we have shown that (joint) hyponormality, while necessary, is not a sufficient condition for lifting [11]. We did this by appealing to commuting 2-variable weighted shifts, for which we have developed new techniques to detect their hyponormality and subnormality. Within the class $\mathfrak{H}_{0}$ of 2-variable weighted shifts $\mathbf{T} \equiv\left(T_{1}, T_{2}\right)$ with commuting subnormal components $T_{1}$ and $T_{2}$, we obtained in [12] a new necessary condition for the existence of a lifting: the Berger measures of horizontal and vertical slices must be linearly ordered with respect to absolute continuity. More recently, we gave in [5] an abstract solution of LPCS, after proving a multivariable version of the Bram-Halmos Criterion [1].

In this paper we develop new tools and techniques, and combine them with those in [11], [12], [13], [5], [9] and [10], to shed light on the spectral theory of 2-variable weighted shifts. As it is well known, the spectral picture of a hyponormal unilateral weighted shift $W_{\alpha}$ is easy to describe: the spectrum is a closed disk of radius $\left\|W_{\alpha}\right\|$, the essential spectrum is the circle of radius $\left\|W_{\alpha}\right\|$, and the Fredholm index is -1 in the open disk. Thus, from a spectral perspective all norm-one hyponormal unilateral weighted shifts are equivalent to the unilateral (unweighted) shift $U_{+}$(which is also subnormal). For subnormal 2-variable weighted shifts the situation is quite different; a complete description of the spectral picture, in the case when the intersection of the boundary of the Taylor spectrum and each coordinate plane is a circle, was given in [9] and [10]. This was done using the groupoid machinery introduced in [16] and [15], and refined in [6]. The presence of the Berger measure was essential in the study of the asymptotic behavior of the weight sequences, and led to concrete results about the various parts of the Taylor spectrum. For hyponormal 2-variable weighted shifts $\mathbf{T} \in \mathfrak{H}_{0}$, however, the study of the spectral properties requires independent techniques, since no Berger measure is present.

In what follows, we present a number of results which highlight the deep differences between 
the single variable case and the 2 -variable case. In Theorem 2.2 we exhibit, for the first time, a sufficient condition that guarantees the disconnectedness of the Taylor essential spectrum, namely, $\left\|W_{\alpha^{(1)}}\right\|<\left\|W_{\alpha^{(0)}}\right\|$, where $W_{\alpha^{(j)}}$ denotes the $j$-th horizontal slice of $T_{1}$; in Example 2.4 we show that this condition can even be present in hyponormal pairs with mutually absolutely continuous horizontal slices $W_{\alpha^{(j)}}(j \geq 1)$. We improve this by showing in Theorem 2.6 that more connected components of the Taylor essential spectrum can be formed, still preserving hyponormality, if we use Bergman-like weighted shifts on each horizontal level of $T_{1}$. This fact is quite surprising, in view of the well known one-variable results. Along the way we prove that, for subnormal 2-variable weighted shifts, the Berger measures of the horizontal slices $\left\{W_{\alpha^{(j)}}\right\}_{j=1}^{\infty}$ are all mutually absolutely continuous and, as a result, $\left\|W_{\alpha^{(j)}}\right\|=\left\|W_{\alpha^{(1)}}\right\|$ (all $j \geq 1$ ). This new necessary condition is easily computable, and complements the previous necessary condition found in [12, Theorem 3.3]. Next, we present an application to single variable operator theory. It is well known that for $T$ a hyponormal operator on $\mathcal{H}, r((T-$ $\left.\lambda)^{-1}\right)=\frac{1}{\operatorname{dist}(\lambda, \sigma(T))}(\lambda \notin \sigma(T))$, where $r$ denotes spectral radius and dist denotes distance. If we substitute the left spectrum for the spectrum, the result is far from obvious; we actually show in Example 2.8 that for $T$ hyponormal, the equality $\left\|\left[(T-\lambda)^{(\ell)}\right]^{-1}\right\|=\frac{1}{\operatorname{dist}\left(\lambda, \sigma_{\ell}(T)\right)}$ may fail, where $\sigma_{\ell}$ denotes the left spectrum and $\left[(T-\lambda)^{(\ell)}\right]^{-1}:=\left[(T-\lambda)^{*}(T-\lambda)\right]^{-1 / 2}$ is the canonical left inverse. Finally, Theorem 2.10 shows that even if we assume that the Berger measures of the shifts associated to horizontal rows and vertical columns are discrete, joint $k$-hyponormality [5] of $\mathbf{T}$ (for an arbitrary $k \geq 1$ ) is not sufficient to force the equality $\left|\sigma_{T e}(\mathbf{T})\right|=\partial\left|\sigma_{T}(\mathbf{T})\right|$. (For compact sets $K \subseteq \mathbb{C}^{2}$ and $L \subseteq \mathbb{R}^{2},|K|:=\left\{\left(\left|z_{1}\right|,\left|z_{2}\right|\right):\left(z_{1}, z_{2}\right) \in K\right\}$ and $\partial L$ denotes the outer boundary of $L$, that is, the boundary of the connected component of $\mathbb{R}^{2} \backslash L$.)

\section{Main Results}

We begin by listing three basic results which are needed in the proofs of Theorems 2.2, 2.6 and 2.10, and Example 2.4.

Lemma 2.1 (i) ([2], [3]) Let $\mathcal{H}_{1}$ and $\mathcal{H}_{2}$ be Hilbert spaces, and let $A_{i} \in \mathcal{L}\left(\mathcal{H}_{1}\right), C_{i} \in \mathcal{L}\left(\mathcal{H}_{2}\right)$ and $B_{i} \in \mathcal{L}\left(\mathcal{H}_{1}, \mathcal{H}_{2}\right),(i=1, \cdots, n)$ be such that $\left(\begin{array}{cc}\mathbf{A} & \mathbf{0} \\ \mathbf{B} & \mathbf{C}\end{array}\right):=\left(\left(\begin{array}{ll}A_{1} & 0 \\ B_{1} & C_{1}\end{array}\right), \ldots,\left(\begin{array}{ll}A_{n} & 0 \\ B_{n} & C_{n}\end{array}\right)\right)$ is commuting. Assume that $\mathbf{A}$ and $\left(\begin{array}{l}\mathbf{A} \mathbf{0} \\ \mathbf{B} \mathbf{C}\end{array}\right)$ are Taylor invertible. Then $\mathbf{C}$ is Taylor invertible. (ii) ([4], [14] and [2]) For $\mathbf{A}$ and $\mathbf{B}$ two commuting n-tuples of bounded operators on Hilbert space, we have $\sigma_{T}(\mathbf{A} \otimes I, I \otimes \mathbf{B})=\sigma_{T}(\mathbf{A}) \times \sigma_{T}(\mathbf{B}), \sigma_{\ell}(\mathbf{A} \otimes I, I \otimes \mathbf{B})=\sigma_{\ell}(\mathbf{A}) \times \sigma_{\ell}(\mathbf{B})$ and $\sigma_{r}(\mathbf{A} \otimes I, I \otimes \mathbf{B})=\sigma_{r}(\mathbf{A}) \times \sigma_{r}(\mathbf{B})$, where $\sigma_{\ell}$ and $\sigma_{r}$ denote the left and right spectra, respectively.

(iii) ([12]) Let $\mu$ be the Berger measure of a subnormal 2-variable weighted shift, and for $j \geq 0$ 
let $\xi_{j}$ be the Berger measure of the associated $j$-th horizontal 1-variable weighted shift $W_{\alpha^{(j)}}$. Then $\xi_{j}=\mu_{j}^{X}$ (the marginal measure of $\mu_{j}$ ), where $d \mu_{j}(s, t):=\frac{1}{\gamma_{0 j}} t^{j} d \mu(s, t)$; more precisely, $d \xi_{j}(s)=\left\{\frac{1}{\gamma_{0 j}} \int_{Y} t^{j} d \Phi_{s}(t)\right\} d \mu^{X}(s)$, where $d \mu(s, t) \equiv d \Phi_{s}(t) d \mu^{X}(s)$ is the disintegration of $\mu$ by vertical slices. A similar result holds for the Berger measure $\eta_{i}$ of the associated $i$-th vertical 1-variable weighted shifts $W_{\beta^{(i)}}(i \geq 0)$.

Theorem 2.2 Let $\mathbf{T}$ be the 2-variable weighted shift given by Figure 1 , let $W_{\alpha^{(j)}}:=\operatorname{shift}\left(\alpha_{0 j}, \alpha_{1 j}, \cdots\right)$ $(j \geq 0), W_{\beta}:=\operatorname{shift}\left(\beta_{0}, \beta_{1}, \cdots\right)$, and let $B:=\beta_{0} \operatorname{diag}\left(1, \frac{\alpha_{01}}{\alpha_{00}}, \cdots\right)$. Assume that $\left\|W_{\alpha^{(j)}}\right\|=$ $\left\|W_{\alpha^{(1)}}\right\|<\left\|W_{\alpha^{(0)}}\right\|($ all $j \geq 2)$. Then $B$ is a compact operator, and $\sigma_{T}(\mathbf{T})=\left(\left\|W_{\alpha^{(1)}}\right\| \cdot \overline{\mathbb{D}} \times\right.$ $\left.\left\|W_{\beta}\right\| \cdot \overline{\mathbb{D}}\right) \cup\left(\left\|W_{\alpha^{(0)}}\right\| \cdot \overline{\mathbb{D}} \times\{0\}\right), \sigma_{T e}(\mathbf{T})=\left(\left\|W_{\alpha^{(1)}}\right\| \cdot \overline{\mathbb{D}} \times\left\|W_{\beta}\right\| \cdot \mathbb{T}\right) \cup\left(\left\|W_{\alpha^{(1)}}\right\| \cdot \mathbb{T} \times\left\|W_{\beta}\right\| \cdot\right.$ $\overline{\mathbb{D}}) \cup\left(\left\|W_{\alpha^{(0)}}\right\| \cdot \mathbb{T} \times\{0\}\right)$. In particular, $\sigma_{T e}(\mathbf{T}) \neq \partial \sigma_{T}(\mathbf{T})$. (Here $\overline{\mathbb{D}}$ denotes the closure of the open unit disk $\mathbb{D}, \mathbb{T}$ the unit circle, and $\left.\partial K:=\left\{\left(z_{1}, z_{2}\right) \in \mathbb{C}^{2}:\left(\left|z_{1}\right|,\left|z_{2}\right|\right) \in \partial|K|\right\}\right)$.

Theorem 2.3 For $i, j \geq 1$, let $W_{\alpha^{(j)}}\left(\operatorname{resp} . W_{\beta^{(i)}}:=\operatorname{shift}\left(\beta_{i 0}, \beta_{i 1}, \cdots\right)\right)$ be the $j$-th horizontal slice (resp. $i$-th vertical slice) of a subnormal 2-variable weighted shift. Then $\left\|W_{\alpha^{(j)}}\right\|=\left\|W_{\alpha^{(1)}}\right\|$ and $\left\|W_{\beta^{(i)}}\right\|=\left\|W_{\beta^{(1)}}\right\|$.

Proof. As a consequence of Lemma 2.1(iii), we see that $\xi_{j} \approx \xi_{1}$ and $\eta_{i} \approx \eta_{1}$ for all $i, j \geq 1$. It follows that $\operatorname{supp}_{j}=\operatorname{supp} \xi_{1}$ and $s u p p \eta_{i}=s u p p \eta_{1}$ for all $i, j \geq 1$. Since the norm of a subnormal unilateral weighted shift always equals the supremum of the support of its Berger measure [1], the result follows.
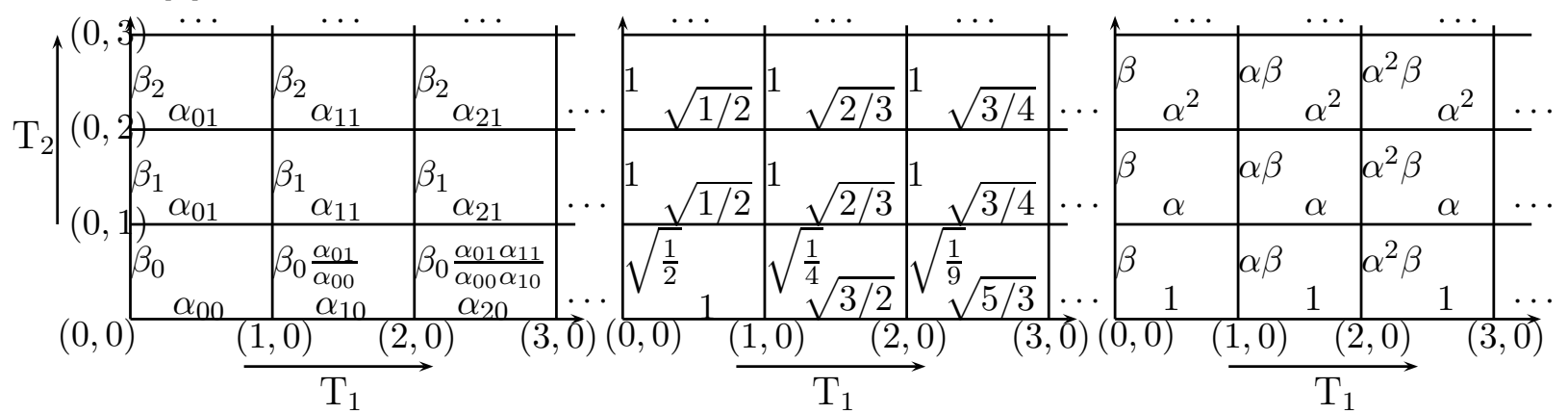

Fig. 1. Weight diagrams of the 2-variable weighted shifts in Theorem 2.2 and Examples 2.4 and 2.7, respectively

Example 2.4 below provides a concrete instance of Theorem 2.2, with all horizontal rows admitting continuous Berger measures. We first need some notation and a few definitions. Let $\mu$ be a Reinhardt measure on $\mathbb{C}^{n}$. The set of bounded point evaluations for $\mu$ is b.p.e $(\mu)=$ $\left\{\lambda \in \mathbb{C}^{n}: p \rightarrow p(\lambda), p \in \mathbb{C}[z]\right.$, extends to a bounded point evaluation from $P^{2}(\mu)$ to $\mathbb{C}$. $\}$. The kernel function associated with $\mu$ is $k(z, w) \equiv k^{(\mu)}(z, w):=\sum_{\alpha \in \mathbb{Z}_{+}^{n}} \frac{z^{\alpha} \bar{w}^{\alpha}}{\left\|z^{\alpha}\right\|^{2}}$, and the set of convergence of $k$ is $\mathcal{C}(k):=\left\{\lambda \in \mathbb{C}^{n}: k(\lambda, \lambda)<\infty\right\}$.

Example 2.4 Let $\mathbf{T} \equiv\left(T_{1}, T_{2}\right)$ be the 2 -variable weighted shift whose weight diagram is given in Figure 1 . Then $\mathbf{T} \in \mathfrak{H}_{0}, \mathbf{T}$ is hyponormal, $\xi_{j} \approx \xi_{1}(j \geq 1), \eta_{i} \approx \eta_{1}(i \geq 0)$ and supp $_{i}=\{0,1\}(i \geq 0)$. However, $\mathbf{T}$ is not subnormal. Moreover $\sigma_{T}(\mathbf{T})=\sigma_{r}(\mathbf{T})=\overline{\mathcal{C}(k)}=$ 
$(\overline{\mathbb{D}} \times \overline{\mathbb{D}}) \cup(\sqrt{2} \cdot \overline{\mathbb{D}} \times\{0\}), \sigma_{T e}(\mathbf{T})=\sigma_{r e}(\mathbf{T})=(\sqrt{2} \mathbb{T}, 0) \cup((\mathbb{T} \times \overline{\mathbb{D}}) \cup(\overline{\mathbb{D}} \times \mathbb{T}))$, and $\partial \sigma_{T}(\mathbf{T}) \neq \sigma_{T e}(\mathbf{T})$ (cf. Figure 2).

Theorem 2.6 below shows that, for every $k \geq 1$, it is possible to create $k$ connected components in the $\sigma_{T e}$ of a 2-variable weighted shift $\mathbf{T}$, while maintaining the hyponormality of $\mathbf{T} \in \mathfrak{H}_{0}$. We recall that for $\ell \geq 1$, the Bergman-like weighted shift on $\ell^{2}\left(\mathbb{Z}_{+}\right)$is $B_{+}^{(\ell)}:=\operatorname{shift}\left(\left\{\sqrt{\ell-\frac{1}{n+2}}\right.\right.$ : $n \geq 0\}$ ); in particular, $B_{+}^{(1)} \equiv B_{+}$is the Bergman shift. Note that $\left\|B_{+}^{(\ell)}\right\|=\sqrt{\ell} \geq 1, B_{+}^{(\ell)}$ is subnormal, with Berger measure $\xi_{\ell}(\ell \geq 1)$, and $d \xi_{1}(s)=d s$ on $[0,1]$ and $d \xi_{2}(s)=\frac{s d s}{\pi \sqrt{2 s-s^{2}}}$ on $[0,2]$ (cf. [7], [13]). The following result is a variation of [13, Theorem 3.14].

Theorem 2.5 For every $k \geq 1$ there exists (i) a family of $\left\{B_{+}^{\left(\ell_{j}\right)}\right\}_{j=0}^{k-1}$ of Bergman-like weighted shifts, and (ii) a subnormal weighted shift $W_{\beta} \equiv \operatorname{shift}\left(\beta_{0}, \beta_{1}, \cdots\right)$ (with $\beta_{n}<\beta_{n+1}$ all $n \geq 0$ ), such that the commuting 2 -variable weighted shift $\mathbf{T}$ with a weight diagram whose first $k$ rows are $B_{+}^{\left(\ell_{0}\right)}, \cdots, B_{+}^{\left(\ell_{k-1}\right)}$, whose remaining rows are all equal to $U_{+}$, and whose 0 -th column is given by $W_{\beta}$, is hyponormal.

Theorem 2.6 Let $\mathbf{T}$ be a hyponormal 2-variable weighted shift satisfying the hypotheses in Theorem 2.5, and let $b^{\left(\ell_{j}\right)}:=\left\|B_{+}^{\left(\ell_{j}\right)}\right\|(j=0, \cdots, k-1)$ and $c:=\left\|W_{\beta}\right\|$. Then $\sigma_{T}(\mathbf{T})=$ $(\overline{\mathbb{D}} \times c \overline{\mathbb{D}}) \cup\left(b^{\left(\ell_{0}\right)} \overline{\mathbb{D}} \times\{0\}\right), \sigma_{T e}(\mathbf{T})=[\overline{\mathbb{D}} \times\{c\}] \cup[\{1\} \times c \overline{\mathbb{D}}] \cup\left[\left(b^{\left(\ell_{0}\right)} \mathbb{T} \cup \cdots \cup b^{\left(\ell_{k-1}\right)} \mathbb{T}\right) \times\{0\}\right]$ (cf. Figure 2).

Example 2.7 shows that, if we don't insist that $\mathbf{T} \in \mathfrak{H}_{0}$ be hyponormal, the Taylor essential spectrum can consist of infinitely many circles converging to a single point. We see in particular that two commuting subnormals do not necessarily give rise to a hyponormal pair.

Example 2.7 Let $\mathbf{T}$ be the 2-variable weighted shift whose weight diagram is given by Figure 1 , where $\alpha<\beta \leq 1$. Then $\mathbf{T} \in \mathfrak{H}_{0}$, with 1-atomic Berger measures for all horizontal and vertical slices. However, $\mathbf{T}$ is not hyponormal. Moreover, $\sigma_{T}(\mathbf{T})$ has empty interior; in fact, $\sigma_{T}(\mathbf{T})=\sigma_{r}(\mathbf{T})=\left[\left(\left|z_{1}\right| \leq 1\right) \times\{0\}\right] \bigcup\left[\{0\} \times\left(\left|z_{2}\right| \leq \beta\right)\right], \sigma_{\ell}(\mathbf{T})=\sigma_{\ell e}(\mathbf{T})=\sigma_{r e}(\mathbf{T}), \sigma_{T e}(\mathbf{T})=$ $\{(0,0)\} \bigcup\left\{\left[\bigcup_{k=0}^{\infty}\left(\left|z_{1}\right|=\alpha^{k}\right)\right] \times\{0\}\right\} \bigcup\left\{\{0\} \times\left[\bigcup \underset{\ell=0}{\infty}\left(\left|z_{2}\right|=\beta \alpha^{\ell}\right)\right]\right\}$ and $\sigma_{\ell}(\mathbf{T})=\sigma_{T e}(\mathbf{T}) \quad(c f$. Figure 2).

We now turn to a simple application of 2 -variable weighted shifts to a single variable problem. Example 2.8 shows that there exists a hyponormal $T \in \mathcal{L}(\mathcal{H})$ with $\left\|(T-\lambda)^{(\ell)^{-1}}\right\| \neq \frac{1}{\operatorname{dist}\left(\lambda, \sigma_{\ell}(T)\right)}$ for some $\lambda \notin \sigma_{\ell}(T)$.

Example 2.8 Let $\mathbf{T}$ be the 2-variable weighted shift whose weight diagram is given by Figure 2, where $a<1$. Then (i) $\mathbf{T}$ is not hyponormal, and $\sigma_{\ell}(\mathbf{T})=\sigma_{\ell e}(\mathbf{T})=(a \mathbb{T} \times \mathbb{T}) \cup(\mathbb{T} \times a \mathbb{T})$; (ii) $T_{1}$ is hyponormal, $\sigma_{\ell}\left(T_{1}\right)=\sigma_{\ell e}\left(T_{1}\right)=a \mathbb{T} \cup \mathbb{T}$ and at least one horizontal slice $W_{\alpha^{(j)}}$ of $T_{1}$ does not satisfy the identity $\left\|\left(W_{\alpha^{(j)}}-\lambda\right)^{(\ell)^{-1}}\right\|=\frac{1}{\operatorname{dist}\left(\lambda, \sigma_{\ell}\left(W_{\left.\left.\alpha^{(j)}\right)\right)}\right.\right.}$ for all $\lambda \in \sigma_{\ell}\left(W_{\alpha^{(j)}}\right)$. 


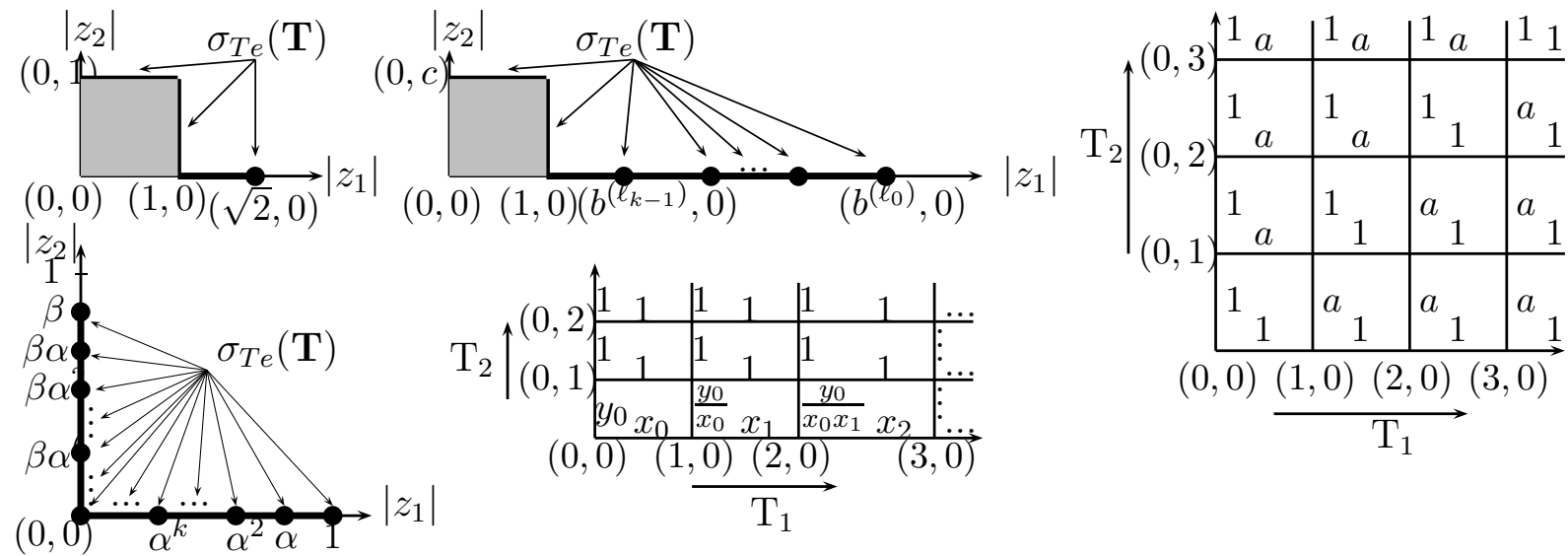

Fig. 2. (top left and center) Spectral pictures in Example 2.4 and Theorem 2.6, respectively; (bottom left) spectral picture in Example 2.7; (top right and bottom center) weight diagrams of the 2-variable weighted shifts in Example 2.8 and Theorem 2.10, respectively

The next result, Theorem 2.10, shows that $k$-hyponormality $(k \geq 1)$ is not sufficient to guarantee $\partial \sigma_{T}(\mathbf{T})=\sigma_{T e}(\mathbf{T})$. We first recall that if $\mathbf{T}$ is a 2-variable weighted shift with weight sequences $\alpha \equiv\left\{\alpha_{\mathbf{m}}\right\}$ and $\beta \equiv\left\{\beta_{\mathbf{m}}\right\}$, then $\mathbf{T}$ is $k$-hyponormal $\Leftrightarrow M_{\mathbf{m}}(k) \geq 0$ for all $\mathbf{m} \in \mathbb{Z}_{+}^{2}$, where $M_{\mathbf{m}}(k)$ is the $(k+1) \times(k+1)$ matrix of moments beginning at $\mathbf{m}([5$, Theorem 3.1]).

Lemma 2.9 (i) For $\kappa>1$, let $x \equiv\left\{x_{n}\right\}_{n=0}^{\infty}$ where $x_{0}:=\sqrt{\frac{1+\kappa}{2}}$ and $x_{n}:=\sqrt{\frac{1+\kappa^{n+1}}{1+\kappa^{n}}}(n \geq 1\}$. Then $W_{\kappa}:=\operatorname{shift}\left(x_{0}, x_{1}, \cdots\right)$ is subnormal, with Berger measure $\xi_{\kappa}=\frac{1}{2}\left(\delta_{1}+\delta_{\kappa}\right)$.

(ii) Given $k \geq 1$ and $m_{1} \geq 0$, consider $M\left(m_{1}\right):=\left(\begin{array}{ccc}\gamma_{m_{1}}\left(W_{\kappa}\right) & \cdots & \gamma_{m_{1}+k}\left(W_{\kappa}\right) \\ \vdots & \ddots & \vdots \\ \gamma_{m_{1}+k}\left(W_{\kappa}\right) & \cdots & \gamma_{m_{1}+2 k}\left(W_{\kappa}\right)\end{array}\right)$ and $\mathbf{1}:=$ $\left(\begin{array}{ccc}1 & \cdots & 1 \\ \vdots & \ddots & \vdots \\ 1 & \cdots & 1\end{array}\right)$. If $0<y_{0}^{2} \leq \frac{1}{2}$ then $M\left(m_{1}\right)-y_{0}^{2} \cdot \mathbf{1} \geq 0$, where $\mathbf{1}$ is $a(k+1) \times(k+1)$ matrix.

Theorem 2.10 Let $\mathbf{T}$ be the 2-variable weighted shift whose weight diagram is given in Figure 2. Given $k \geq 1, \kappa>1$ and $W_{\kappa}$, there exists $0<y_{0} \leq \sqrt{\frac{1}{2}}$ which makes $\mathbf{T} k$-hyponormal. Moreover, $\sigma_{T}(\mathbf{T})=\sigma_{r}(\mathbf{T})=(\overline{\mathbb{D}} \times \overline{\mathbb{D}}) \cup(\sqrt{\kappa} \cdot \overline{\mathbb{D}} \times\{0\}), \sigma_{T e}(\mathbf{T})=\sigma_{r e}(\mathbf{T})=(\sqrt{\kappa} \mathbb{T} \times 0) \cup((\mathbb{T} \times$ $\overline{\mathbb{D}}) \cup(\overline{\mathbb{D}} \times \mathbb{T})$ ), and $\partial \sigma_{T}(\mathbf{T}) \neq \sigma_{T e}(\mathbf{T})$.

Sketch of Proof. Observe that the restriction of $\mathbf{T}$ to $\vee\left\{e_{\left(m_{1}, m_{2}\right)}: m_{2} \geq 1\right\}$ is unitarily equivalent to $\left(I \otimes U_{+}, U_{+} \otimes I\right)$. We then apply $\left(\left[5\right.\right.$, Theorem 3.1]) to $\mathbf{m}:=\left(m_{1}, 0\right)$, with $m_{1} \geq 0$. We have $M_{\left(m_{1}, 0\right)}(k) \geq 0$ (all $\left.k \geq 1\right) \Leftrightarrow M\left(m_{1}\right)-y_{0}^{2} \cdot 1 \geq 0$. We then use the moments associated with $\mathbf{T}$, matrix row operations, Choleski's algorithm, and a choice of $y_{0} \leq \sqrt{\frac{1}{2}}$, together with Lemma $2.9(\mathrm{i})$, to show that $\mathbf{T}$ is $k$-hyponormal. To calculate $\sigma_{T}(\mathbf{T})$ 
and $\sigma_{T e}(\mathbf{T})$, we use Lemma 2.1, the projection property for the Taylor spectrum, and direct verification of the exactness of the Koszul complex associated with $\mathbf{T}$ at the middle stage, using the technique in [8, Corollary 4.3(ii)].

Remark 2.11 Careful analysis of the proof of Theorem 2.10 reveals that for $k \geq 1$ and

$y_{0} \leq \sqrt{\frac{1}{2}}, \mathbf{T}$ is k-hyponormal. Thus, $\mathbf{T}$ is indeed subnormal in the interval $\left(0, \sqrt{\frac{1}{2}}\right]$, and its Berger measure is $\mu=y_{0}^{2} \delta_{1} \times \delta_{1}+\left(\xi_{\kappa}-y_{0}^{2} \delta_{1}\right) \times \delta_{0}$.

\section{References}

[1] J. Conway, The Theory of Subnormal Operators, Mathematical Surveys and Monographs, vol. 36, Amer. Math. Soc., Providence, 1991.

[2] R. Curto, Spectral theory of elementary operators, in Elementary Operators and Applications, M. Mathieu, ed., World Sci. Publishing, River Edge, NJ, 1992; pp. 3-52.

[3] R. Curto, Applications of several complex variables to multiparameter spectral theory, Surveys of Some Recent Results in Operator Theory, Vol. II, J.B. Conway and B.B. Morrel, editors, Longman Publishing Co., London (1988); pp. 25-90.

[4] R. Curto and L. Fialkow, The spectral picture of $\left(L_{A}, R_{B}\right)$, J. Funct. Anal. 71(1987), 371-392.

[5] R. Curto, S.H. Lee and J. Yoon, $k$-hyponormality of multivariable weighted shifts, J. Funct. Anal. 15(2005) 462-480.

[6] R. Curto and P. S. Muhly, C*-algebras of multiplication operators on Bergman spaces, J. Funct. Anal. 64(1985), 315-329.

[7] R. Curto, Y.T. Poon and J. Yoon, The class of the Bergman-like weighted shifts, J. Math. Anal. Appl. 308(2005) 334-342.

[8] R. Curto and N. Salinas, Spectral properties of cyclic subnormal m-tuples, American J. Math. 107(1985), 113-138.

[9] R. Curto and K. Yan, The spectral picture of Reinhardt measures, J. Funct. Anal. 131 (1995), no. 2, 279-301.

[10] R. Curto and K. Yan, Spectral theory of Reinhardt measures, Bull. Amer. Math. Soc. (N.S.) 24 (1991), no.2, 379-385.

[11] R. Curto and J. Yoon, Jointly hyponormal pairs of commuting subnormal operators need not be jointly subnormal, Trans. Amer. Math. Soc. 358(2006), 5139-5159.

[12] R. Curto and J. Yoon, Disintegration of measure techniques for commuting multivariable weighted shifts, Proc. London Math. Soc. 92(2006) 381-402. 
[13] R. Curto and J. Yoon, Propagation Phenomena for hyponormal 2-variable weighted shifts, $J$. Operator Theory, to appear.

[14] L.A. Fialkow, Spectral properties of elementary operators II, Trans. Amer. Math. Soc. 290 (1985), 415-429.

[15] P. Muhly and J. N. Renault, $C^{*}$-algebras of multivariable Wiener-Hopf operators, Trans. Amer. Math. Soc. 274(1982), 1-44.

[16] J. N. Renault, A groupoid approach to $C^{*}$-algebras, Lecture Notes in Mathematics, 793. Springer, Berlin, 1980. 